\title{
Consumer perception of natural hot-pepper cheeses
}

\author{
C. M. Racette (D) and M. A. Drake* (1) \\ Southeast Dairy Foods Research Center, Department of Food, Bioprocessing, and Nutrition Sciences, North Carolina State University, \\ Raleigh 27606
}

\begin{abstract}
Hot-pepper cheese (HPC) is a growing category of flavored natural cheese. The objective of this study was to evaluate consumer perception of HPC using a combination of quantitative survey methods and consumer evaluation of HPC. An online survey $(\mathrm{n}=510)$ was conducted to understand drivers of purchase for the HPC category. Consumers of HPC answered maximum difference exercises and an adaptive choice-based conjoint activity focused on HPC attributes. Subsequently, natural HPC were manufactured in duplicate with 5 different hot-pepper blends with a range of heat intensities and distinct color differences. Trained panel profiling and consumer-acceptance testing ( $\mathrm{n}=194$ consumers) were conducted on the cheeses. Three clusters of consumers were identified from the online survey. Cluster $1(\mathrm{n}=175)$ were traditional HPC consumers, and cluster $2(\mathrm{n}=152)$ preferred milder HPC. Cluster $3(\mathrm{n}=183)$ showed preference for spicier HPC as well as novel HPC, such as those made with habanero peppers or white Cheddar cheese. Conceptually, the overall ideal HPC was a Monterey Jack with medium-sized, multicolored pieces of jalapeno peppers and a medium heat and spiciness. Heat and spiciness intensity and type of cheese were the most important attributes. The 5 HPC used in consumer testing had a distinct range (low to high) of hot-pepper burn and heat intensity by trained panel profiling. Consumer overall liking increased as hot-pepper burn and heat intensity increased to a certain point, indicating HPC consumers may have an optimal point for heat and spiciness in HPC. Consumers also preferred HPC with multicolored pepper pieces over those with a single pepper color, consistent with survey results. Consumers who self-reported that they prefer mild- or medium-spicy foods (mild consumers) preferred HPC that were less intense in hot-pepper burn than consumers who self-reported preference for hot or spicy foods (hot consumers). Most HPC consum-
\end{abstract}

Received May 31, 2021.

Accepted October 1, 2021.

*Corresponding author: mdrake@ncsu.edu ers preferred HPC with higher heat intensity and were also motivated by visual characteristics of HPC.

Key words: hot-pepper cheese, consumer, liking

\section{INTRODUCTION}

Consumption of cheese has been on the rise in the United States since 2000. In 2019, the USDA reported American cheese consumption as $15.5 \mathrm{lb} /$ capita, compared with $12.7 \mathrm{lb} /$ capita in 2000 (Shahbandeh, 2020). In August 2020, Mintel surveyed consumers who selfreported eating cheese more often than they did in the last year to provide more context to these trends. Consumers $(\mathrm{n}=479)$ indicated that they snacked on cheese more often, used it in more meals, and consumed more types of cheese. Cheese consumers also reported that unique flavors and all-natural ingredients were motivating factors for purchasing and consuming a new cheese (Kamp, 2020). With increasing consumer interest in innovative and diverse cheeses, there has been a subsequent increase in the flavored-cheese category, including cheeses that contain hot peppers. Forty-one percent of consumers in a Mintel study from November 2019 to June 2020 ( $\mathrm{n}=9$,009) indicated that they purchased and consumed Pepper Jack cheese, compared with 39\% from August 2019 Mintel data ( $=1,401$ ) (Kamp, 2020). Traditionally, hot-pepper cheese (HPC) has been constrained to Pepper Jack, which refers to Monterey Jack cheese with sweet peppers, habaneros, or jalapenos. However, in recent years, the category has grown to include different cheese types, such as Cheddar, Gouda, and Colby Jack, as well as new pepper types.

Hot-pepper cheeses are made by adding pepper pieces or other flavorants to the cheese curds before pressing, resulting in a cheese that has pepper pieces dispersed throughout the loaf. To our knowledge, few studies have specifically addressed the sensory perception of hot-pepper cheese. Some studies have evaluated the perception of capsaicin burn in cheese matrices. Carden et al. (1999) studied perception of heat in cheese sauces with varying fat and fat mimetic levels using a trained panel and found that reducing fat content increased perceived burn from capsaicin. Capsaicin is lipophilic 
and is more soluble in fat (Reyes-Escogido et al., 2011). As such, sensory perception of capsaicin burn would be expected to be higher in reduced-fat versions of foods. Other researchers have used paneer cheese as a matrix to study perception of capsaicin burn to correlate consumer results with electronic-tongue analysis (Schlossareck and Ross, 2019). Other researchers have used consumer-acceptance testing and survey methods to evaluate consumer perception of different options in the flavored and specialty cheese categories, such as raw milk Gouda and smoked cheeses (Colonna et al., 2011; Del Toro-Gipson et al., 2021).

Several studies have used quantitative consumer research methodologies to assess consumer perception of food products. Kano analysis was originally developed by Noriaki Kano for use in assessing consumer satisfaction in quality assurance (Kano et al., 1984; Rotar and Kozar, 2017). This technique uses a series of questions regarding the presence or absence of features to assess consumer basic needs for a product along with the characteristics that excite them. Consumer responses in a Kano exercise are grouped into 1 of 3 categories as follows: basic needs, performance needs, and delight needs. These categories are further broken down into attractive, indifferent, must-be, performance (one-dimensional), questionable, or reverse characteristics. Performance characteristics follow the "more is better rule," whereas must-be characteristics are those that consumers consider basic needs. Attractive characteristics are unexpected by the consumer but result in increased satisfaction with the product, whereas reverse characteristics are those that the consumer would prefer not to see in a product (Rotar and Kozar, 2017). Kano analysis can be applied to a wide range of products, but only a small number of studies have applied this technique to the food and restaurant industry (Ponnam et al., 2011; Chen, 2012; Kim et al., 2013; Djekic et al., 2020). Kim et al. (2013) used Kano analysis to evaluate the effect of chocolate milk attributes on consumer satisfaction or dissatisfaction and found that distinct groups of consumers responded differently to fat-content levels (fat free, $1 \%$ fat, $2 \%$ fat, and whole milk).

Conjoint analysis is an effective tool in guiding product development by quantifying consumer preferences for food product attributes (Moskowitz, 2001). This method uses a series of complete product profiles to assess which attributes and attribute levels consumers place value on. This approach has been used to identify key attributes for many foods including sour cream, chocolate milk, protein beverages, and bacon (Jervis et al., 2012; Kim et al., 2013; Oltman et al., 2015; McLean et al., 2017). Other studies have used maximum difference (MXD) scaling, also known as best-worst scal- ing, to assess the importance that consumers place on individual product attributes and label claims. This methodology asks consumers to choose the "best" and "worst" items from a list of provided options to conduct simultaneous paired comparisons on a long list of items (Orme, 2006). Schiano et al. (2020) used a series of MXD exercises to assess consumer perception of the sustainability of dairy products and plant-based dairy alternatives and rank the importance of dairy attributes in terms of sustainability. Del Toro-Gipson et al. (2021) used a combination of methods including focus groups, 2 online surveys, and a consumer-acceptance test to evaluate perception of smoked Cheddar cheeses. Results of these studies provide actionable insights for food manufacturers and provide further guidance to sensory researchers on the use of quantitative consumer research methods. With increasing variety in the category, it is important for cheese manufacturers to understand the desirable sensory properties of hot-pepper cheese to facilitate product development and strategic positioning. The purpose of this study was to gain a better understanding of consumer perception of HPC.

\section{MATERIALS AND METHODS}

\section{Experimental Overview}

An online survey using ethnographic questions, MXD scaling, a Kano exercise, and an adaptive choice-based conjoint (ACBC) exercise was conducted. This survey was conducted to establish consumer familiarity with and preference for attributes of HPC, including extrinsic and intrinsic characteristics. Following completion of the survey, 5 natural Monterey Jack cheeses with a range of pepper colors and burn intensities were manufactured in duplicate. Descriptive analysis and a consumer-acceptance test were conducted on the cheeses.

\section{Participants}

All testing was conducted in compliance with North Carolina State University Institutional Review Board regulations. All participants were contacted using an online database of $>10,000$ consumers from the greater Raleigh-Durham-Chapel Hill North Carolina area maintained by the Sensory Service Center at North Carolina State University. Consumers who reported that they did not have any food allergies, intolerances, or dietary restrictions, and who indicated that they purchased and consumed HPC could qualify for this study. Participants completed an electronic informed consent before beginning participation in study activities. Additional qualification criteria for the survey and consumer-acceptance test are subsequently specified. 


\section{Online Survey}

The objective of the online survey was to establish a baseline of consumer familiarity with attributes of HPC, including its visual appearance, spiciness, and other intrinsic characteristics. The online survey was developed using Lighthouse Studio (Sawtooth software version 9.8.1). The survey was uploaded to the database maintained by the Sensory Service Center at North Carolina State University. Participants who were $18 \mathrm{yr}$ of age or older were able to enter the survey $(\mathrm{n}=1,386)$. Participants who indicated that they purchased HPC ( $\mathrm{n}=$ 510) first completed a series of psychographic questions, including familiarity with HPC characteristics and purchase and consumption frequency of HPC. Familiarity was measured using a 5 -point scale where $1=$ not at all familiar, 2 = slightly familiar, $3=$ somewhat familiar, 4 $=$ very familiar, and $5=$ extremely familiar. Next, they answered questions about their ideal balance of pepper flavor and cheese flavor in HPC using a semantic differential (sliding scale) question where $-100=$ only cheese flavor, $0=$ both cheese and hot-pepper flavor, and $100=$ only hot-pepper flavor. Following the semantic differential question, survey participants completed an MXD exercise on HPC characteristics, followed by a series of questions for Kano analysis. Consumers were then asked to indicate which extrinsic and intrinsic factors of HPC influenced their purchase decisions using a check-all-that-apply (CATA) list. Participants finished the survey with an ACBC exercise.

The MXD exercise consisted of 14 different purchase factors for HPC, including extrinsic qualities such as all-natural and organic labels, and intrinsic factors such as type of pepper used, type of cheese used, and spiciness intensity. Respondents were asked to indicate which item was "most important" and which item was "least important" from a list of attributes. Each respondent completed 15 sets of best-worst questions with 4 items in each set. These questions were asked to evaluate which attributes consumers placed the most importance on when selecting an HPC to purchase and consume and to gain a better understanding of the role of visual appearance of pepper cheese in purchase decisions.

The Kano exercise consisted of 56 total statements regarding HPC attributes, including 28 "positive" statements such as "is fortified with vitamins" and 28 directly opposite "negative" statements such as "is not fortified with vitamins." Consumers were asked to rate each statement on a 5-point scale where $1=\mathrm{I}$ enjoy it that way, $2=\mathrm{I}$ expect it that way, $3=\mathrm{I}$ am neutral, 4 $=\mathrm{I}$ dislike it, but I can live with it, and $5=\mathrm{I}$ cannot accept it (Anonymous, 2021; Rotar and Kozar, 2017).
Several types of conjoint analysis have been developed, including adaptive conjoint analysis, choicebased conjoint analysis, and ACBC. We used ACBC in this study as it is less time-intensive and fatiguing for participants than adaptive conjoint analysis and provides more robust estimation of part-worth utilities than choice-based conjoint analysis (Orme, 2006; Jervis et al., 2012). Previous research has shown that ACBC can be used to effectively assess consumer perception of cheese and other dairy products (Harwood and Drake, 2018; Rizzo et al., 2020; Del Toro-Gipson et al., 2021). The ACBC included a build-your-own (BYO) exercise followed by 8 screening tasks with 4 concepts per screening task and concluded with a choice tournament with 3 concepts per choice task. During the BYO portion of the exercise, survey participants used a provided list of attributes and levels to build their ideal HPC. The ACBC design was comprised of the following 6 attributes: type of cheese, type of pepper, size of pepper pieces, amount of pepper pieces, pepper color, and spiciness intensity with levels within each attribute. Attributes related to visual characteristics (type of cheese, size of pepper pieces, amount of pepper pieces and pepper color) were also displayed in a graphic format in the ACBC. Graphics were generated using Adobe Illustrator (v23.0.3, Adobe Inc.) for each unique combination of levels (108 graphics in total) and were displayed during the BYO exercise and with each concept during the screenings tasks and the choice tournament. Participants who completed the entire survey were entered into a drawing for 1 of 10 gift cards to a local retailer.

\section{Evaluation of Natural HPC}

Following completion of the online survey, 5 natural HPC with a range of pepper burn intensities and visual characteristics were manufactured by an industry partner (Twin Falls, ID). Two lots of full-fat pasteurized milk Monterey Jack cheese were produced $(25 \mathrm{~kg}$ of cheese per lot). Diced peppers were sourced from commercial sources and included hatch peppers, red and green jalapenos, and orange habaneros to generate a range of pepper color and pepper burn (Table 1). Pepper combinations were premixed, added to drained cheese curds (3.5\% wt/wt), and mixed thoroughly. Cheese curds with the pepper inclusions were transferred into stainless steel hoops lined in cheese cloth and pressed at $414 \mathrm{kPa}$ for $30 \mathrm{~min}$. Cheese blocks (5 $\mathrm{kg}$ ) were cooled using forced-air coolers at $5^{\circ} \mathrm{C}$. The duplicate cheese lots were manufactured within 1 wk of each other. Cheeses were shipped by overnight carrier to Raleigh, North Carolina, at 30 d. Trained panel and 
Table 1. Pepper formulations used for manufacture of hot-pepper cheeses ${ }^{1}$

\begin{tabular}{|c|c|c|c|}
\hline Prototype & Pepper type & Dice size & Ratio \\
\hline Formulation 2 & $\begin{array}{l}\text { Green hatch peppers } \\
\text { Red jalapenos }\end{array}$ & $\begin{array}{l}\text { hatch } 3 / 8^{\prime \prime} \\
\text { jalapeno } 1 / 8^{\prime \prime}\end{array}$ & $50 \%$ hatch peppers, $50 \%$ jalapenos \\
\hline Formulation 4 & $\begin{array}{l}\text { Red jalapenos } \\
\text { Green jalapenos } \\
\text { Orange habaneros }\end{array}$ & $1 / 8^{\prime \prime}$ & $\begin{array}{l}27 \% \text { red jalapenos, } 73 \% \text { green jalapenos } \\
70 \% \text { jalapeno pepper mix, } 30 \% \text { habanero }\end{array}$ \\
\hline
\end{tabular}

${ }^{1}$ Pepper pieces were sourced from Mizkan and Edco Food Products.

consumer testing occurred within a 2 -wk period when the cheeses were $50 \mathrm{~d}$ old.

\section{Descriptive Analysis}

Seven trained panelists ( 5 women, 2 men, ages $22-54$ $\mathrm{yr}$ ), each with at least $60 \mathrm{~h}$ of previous experience with the descriptive analysis of cheese, evaluated HPC cheeses from each lot in duplicate. A 0-to- 15 point universal intensity scale consistent with the Spectrum descriptive analysis method (Meilgaard et al., 2007) was used for analysis. An established cheese lexicon was applied with 2 additional attributes, pepper aromatic and hot-pepper burn (Drake et al., 2001). Hot-pepper burn is also referred to as pungency or burn intensity (Cliff and Heymann, 1992; Allison et al., 1999; Guzmán and Bosland, 2017) and is separate from the aromatics imparted by hot peppers. Pepper aromatic was defined for this study as aromatics associated with freshly chopped peppers (chopped green and red bell peppers were used as an example), whereas hot-pepper burn was defined as the specific chemesthetic sensation of burning associated with hot peppers (cinnamon oil and capsaicin were used as examples). Each cheese was cut into a $3-\mathrm{cm}$ cube and placed into a lidded $60-\mathrm{mL}$ soufflé cup with a randomly generated 3 -digit code and tempered to $15^{\circ} \mathrm{C}$. Samples were prepared with overhead lights off to prevent light oxidation. Paper ballots were used for data collection. A 10-min rest was enforced between samples. Panelists evaluated cheeses in a randomized balanced order and were instructed to expectorate samples, rinse their mouths with deionized water, and take a bite of unsalted saltine cracker between cheeses. Each panelist evaluated each lot in duplicate in separate sessions.

\section{Consumer Testing}

A consumer-acceptance test modified for COVID-19 safety was conducted in October 2020 using consumers recruited from the database maintained by the Sen- sory Service Center. Consumers aged 18 to 64 yr that purchased and consumed HPC at least once a month were recruited. Data were collected using an electronic ballot using Compusense Cloud software (Compusense Inc.). Cheeses from both lots were pooled for consumer testing as descriptive analysis results showed the 2 lots were not significantly different in sensory profile $(P>$ 0.05 ; results not shown). Each consumer was provided with 4 of the 5 cheeses in a balanced incomplete block design. A total of 200 consumers were recruited to provide at least 160 to 180 completed ballots on each of the 5 cheeses. Each cheese was cut into a $3-\mathrm{cm}$ cube and placed into a lidded $60-\mathrm{mL}$ soufflé cup with a randomly generated 3-digit code. Samples were prepared with overhead lights off to prevent light oxidation. For this test, qualified consumers picked up a thermal bag (ULINE) with coded samples, a Ziploc bag of unsalted saltines, and a commercial bottle of spring water. Consumers were instructed to take the samples home and then to click on the provided hyperlink to complete the test within $4 \mathrm{~h}$ of pickup.

Once consumers clicked on the ballot link and initiated the test, they were asked to rank the cheeses in order of how much they liked their appearance $(1=$ most liked, $4=$ least liked), by the amount of pepper pieces they could see in the sample $(1=$ most pepper pieces, 4 $=$ least pepper pieces), and how spicy they thought the cheeses would be based only on appearance $(1=$ spiciest or hottest and $4=$ least spicy or mildest). Following the ranking questions, cheeses were evaluated monadically in a randomized order. For each cheese, consumers were first asked to evaluate appearance liking (no tasting). Liking questions were evaluated using a 9-point hedonic scale where $1=$ dislike extremely and $9=$ like extremely. Next, consumers evaluated the amount of pepper pieces using a 5-point just-about-right (JAR) scale where 1 and $2=$ not enough pepper pieces, 3 $=$ just about right, and 4 and $5=$ too many pepper pieces. Consumers then evaluated their liking of the color of the pepper pieces. Next, consumers indicated 
overall liking. Next, they answered spiciness liking and a spiciness JAR question where 1 and $2=$ not spicy enough, $3=$ just about right, and 4 and $5=$ too spicy. Cheese flavor and pepper flavor liking were evaluated next, followed by a flavor balance JAR where 1 and $2=$ too much cheese flavor, $3=$ just about right, and 4 and $5=$ too much pepper flavor. Panelists then evaluated saltiness liking and saltiness JAR using a 5-point scale in which 1 and 2 = not salty enough, 3 = just about right, and 4 and $5=$ too salty. Following flavor questions, panelists evaluated the texture of the cheeses, including liking and a texture JAR question in which 1 and $2=$ too soft, $3=$ just about right, and 4 and 5 $=$ too firm. Finally, consumers indicated their purchase intent using a 5-point scale in which $1=$ definitely will not buy, $2=$ probably will not buy, $3=$ might or might not buy, $4=$ probably will buy and $5=$ definitely will buy. Participants were required to rest for $10 \mathrm{~min}$ and cleanse their palates with unsalted crackers between cheeses. After tasting all 4 cheeses, participants were asked to rank the cheeses in order of preference $(1=$ most preferred and $4=$ least preferred). Participants finished the test by answering demographic questions, including their personal spice level preference, frequency of HPC consumption, pepper type familiarity, and factors of purchase and consumption for HPC. After completing the test, participants were compensated with a $\$ 20$ gift card to a local store.

\section{Statistical Analysis}

The MaxDiff and ACBC scores from the online survey were evaluated using hierarchical Bayesian regression using Lighthouse Studio. All other analyses were performed with XLSTAT (version 2019.3.1, Addinsoft) at $95 \%$ confidence $(P<0.05)$. Cluster analysis of individual importance scores was performed using Euclidean distances and Ward's linkage to categorize respondents with similar answers into separate groups. One-way ANOVA with means separation (Fisher's LSD with a Bonferroni correction) was performed on the trained panel attribute intensities and consumer liking data. Consumer JAR questions were analyzed with penalty analysis and chi-squared analysis with the Marasculio procedure for multiple comparisons. Ranking data from consumer testing was analyzed using Kruskal-Wallis testing with Dunn's multiple comparison test.

\section{RESULTS AND DISCUSSION}

\section{Online Survey Participant Demographics}

A total of $\mathrm{n}=1,386$ consumers entered the online survey. Of these participants, $\mathrm{n}=510$ met the criteria for participation previously described. Participants were mostly women ( $69 \%$ vs. $31 \%$ men). Survey participants represented a range of ages, with $20 \%$ of participants aged 18 to 24 yr old, $30 \%$ aged 25 to 34 yr old, $20 \%$ aged 35 to 44 yr old, $14 \%$ aged 45 to 54 yr old, and $16 \%$ aged 55 yr or older. Most survey participants $(78 \%)$ self-identified as white/Caucasian, $10 \%$ identified as Asian/Indian, and 9\% identified as Black/ African American. Consumers indicated high frequency of spicy food consumption, with $65 \%$ reporting consuming spicy foods at least once per week, $25 \%$ reporting spicy food consumption once every $2 \mathrm{wk}$, and $10 \%$ reporting occasional (around once per month) spicy food consumption. Almost half $(47 \%)$ of consumers preferred medium-spicy products, $11 \%$ preferred mild, $37 \%$ preferred hot, and 6\% preferred extremely hot. A similar distribution of preference for spice and burn was documented in a series of online surveys conducted in 12 countries with $\mathrm{n}=500$ participants each, with $40 \%$ of consumers self-reporting as medium users, $28 \%$ self-reporting as hot users, and 7\% self-reporting as extremely hot users (Anonymous, 2019). Participants indicated the highest familiarity with jalapeno peppers, returning a 4.5 overall score on a 5-point familiarity scale where $1=$ not at all familiar and $5=$ extremely familiar. Participants were also familiar with habaneros (3.9), chipotles (3.7), and poblanos (3.5). However, consumers indicated limited familiarity with Carolina Reaper peppers (2.6), Hatch chiles (2.4), and Scotch Bonnet peppers (2.3). Most survey participants were moderate HPC users, with $26 \%$ reporting HPC consumption about once per week, $25 \%$ about once every 2 wk, and $23 \%$ about once per month. Twelve percent of survey participants indicated they consume HPC about 3 to 5 times per week. Overall, consumers indicated they prefer a balance of hot-pepper and cheese flavor in HPC, returning a score of 2.96 on a sliding scale where $-100=$ only cheese flavor and $100=$ only hotpepper flavor. As with any survey, a limitation of the results is that they represent the participants within the database. Most the participants lived within the state of North Carolina, but participants from 26 other states within the United States participated (results not shown). Future work could address a wider sampling of consumers to confirm the generalization of these results.

Consumers indicated familiarity with HPC (4.0 on a 5-point familiarity scale), but were less familiar with different cheeses (2.8) and peppers (2.7) used in HPC. Consumers also indicated limited familiarity with the spiciness intensity of different peppers (3.3). Price and type of cheese were the most-checked CATA attributes when consumers were asked to indicate which factors influenced their decision to purchase HPC (84 and 78\%, 
respectively). Consumers also indicated that appearance of cheese $(73 \%)$, spiciness intensity $(67 \%)$, and type of pepper (52\%) were influential. Extrinsic factors, such as sustainably sourced (18\%), ethical treatment of cows $(18 \%)$, organic $(16 \%)$, and GMO-free $(16 \%)$ were less important in purchase decisions.

\section{MXD Exercise}

The MXD exercises generate importance scores, which indicate the amount of value that consumers place on a product attribute when making purchase decisions. Higher importance scores reflect a greater degree of importance for an attribute (Orme, 2006). The MXD results from the HPC online survey showed that spiciness intensity and type of cheese, followed by price, were the most important attributes of HPC to consumers (Figure 1). Spiciness intensity and type of cheese were not different from each other $(P>0.05)$ and were the most important attributes for HPC purchase $(P<0.05)$. Consumers indicated that extrinsic characteristics such as organic labels, brand name, GMO-free labels, and packaging were least important to them, consistent with their CATA responses earlier in the survey. These results align with those observed by Del Toro-Gipson et al. (2021), who observed that consumers valued type of cheese and type of smoke over label claims and other extrinsic characteristics when purchasing smoked cheeses.

\section{Kano Analysis}

In Kano analysis, consumer responses to both the presence and absence of a product feature are used to categorize features into 1 of 6 categories as follows: attractive, indifferent, must-be, performance, questionable, or reverse (Rotar and Kozar, 2017). Most consumers were indifferent about HPC characteristics, with low price being the only characteristic that was perceived as attractive overall (data not shown). Price is gener- ally a universally attractive attribute for food (McLean et al., 2017; Harwood and Drake, 2018; Speight et al., 2019). Kim et al. (2013) also observed that chocolate milk consumers were indifferent to most chocolate milk attributes in a Kano exercise, but found fat content attractive (Kim et al., 2013). "DHA-fortified," "name brand," and "fortified with vitamins" received the highest percentage of neutral or indifferent responses $(83.9,80.0$, and $78.8 \%$ respectively), which might be expected given that these are not attributes or labels commonly associated with natural cheeses of any kind. However, other HPC characteristics received lower frequencies of indifferent responses and higher frequencies in the attractive, performance, and must-be categories, indicating there may be groups of consumers who perceive these characteristics differently. "Contains pepper pieces" was an indifferent characteristic for only $35.1 \%$ of respondents, with $21.4 \%$ indicating pepper pieces in HPC are attractive, $20.4 \%$ indicating pepper pieces are a performance attribute, and $20.4 \%$ indicating that pepper pieces are a must-be attribute for HPC. Similarly, "locally produced" and "contains more than one pepper type" were perceived as attractive by several consumers (41.2 and $36.9 \%$ respectively). "Contains natural preservatives to extend shelf life" and "intense spiciness" were also perceived as attractive by a subset of consumers (29.8 and $25.9 \%$ respectively).

Responses to Kano questions were segmented using consumer self-reported preferred spice or heat level in spicy foods to evaluate if spice preferences influenced consumer satisfaction characteristics in HPC. Mild consumers $(\mathrm{n}=55)$ found "mild spiciness" and "low price" attractive, but were indifferent toward all other HPC characteristics. Medium consumers ( $\mathrm{n}=238)$ found "low price" attractive but were indifferent toward other characteristics. Hot consumers $(\mathrm{n}=188)$ were indifferent to all characteristics, but the same number of individuals in this group found "intense spiciness" attractive as the number of those who were indifferent to this characteristic. Extra hot consumers $(\mathrm{n}=29)$

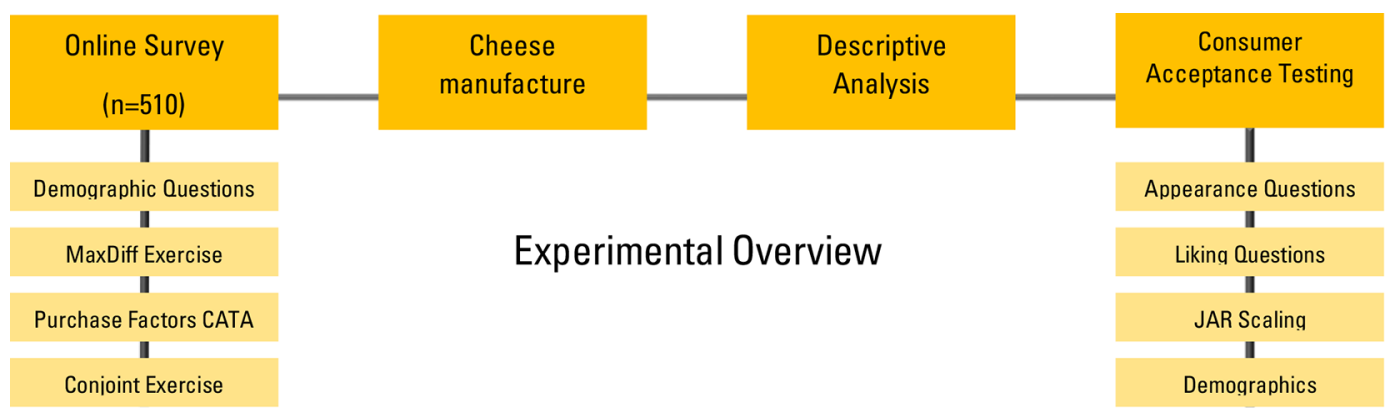

Figure 1. Overview of experimental design. JAR = just-about-right; CATA = check-all-that-apply. 


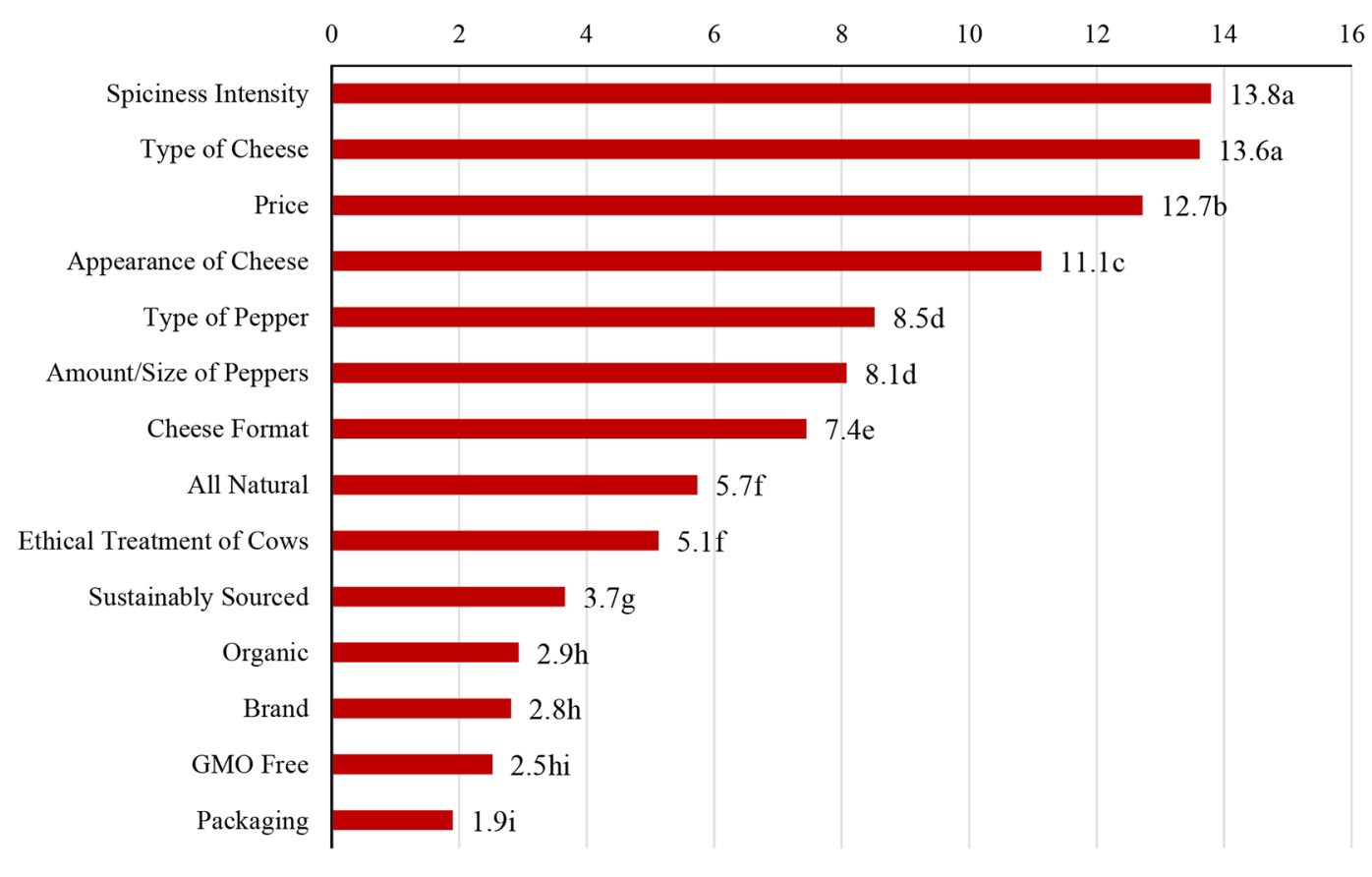

MXD score

Figure 2. Mean maximum difference (MXD) scores for important hot-pepper cheese characteristics $(\mathrm{n}=510)$. Means followed by different letters $(\mathrm{a}-\mathrm{i})$ are significantly different $(P<0.05)$. The MXD scores have been rescaled to sum to 100 . A higher score indicates a more important attribute.

indicated that "contains more than one pepper type, "intense spiciness," and "low price" were attractive, and an equal number of consumers in this group also found "melts well" and "locally produced" attractive.

\section{ACBC Exercise}

Conjoint exercises produce values known as utility scores, which are derived from hierarchical Bayesian estimation analysis. These values are zero-centered and rescaled to allow all values to sum to 100 . More positive scores are representative of more appealing levels within an attribute, whereas more negative scores represent less appealing characteristics (Orme, 2006). Overall, the ideal HPC for consumers was a Monterey Jack cheese with medium-sized, multicolored (red and green) pieces of jalapeno peppers at a medium frequency and a medium heat and burn intensity (Figure 2). Monterey Jack cheese (42.9) and white Cheddar (42.5) received similar utility scores $(P<0.05)$, indicating that either of these cheese options may be appealing to HPC consumers. In contrast, survey participants returned the lowest utility score for orange Cheddar $(-66.5)$ out of all variables presented $(P<0.05)$, which suggested that orange Cheddar as a cheese for HPC was less appealing to consumers. It has been previously observed that consumers value intrinsic characteristics of flavored cheeses; smoked Cheddar consumers placed importance on smoking method, smoke intensity, type of wood, and type of cheese (Del Toro-Gipson et al., 2021).

Conjoint results were analyzed using agglomerative hierarchical clustering and 3 distinct groups of consumers with different preferences for HPC were identified (Figure 2). Age and gender demographic distributions were consistent among all 3 groups $(P>0.05)$. Cluster $1(\mathrm{n}=175)$ designated the traditional consumers, who indicated their ideal HPC was a Monterey Jack with a high frequency of medium-sized, multicolored jalapeno pieces and a medium spice and burn intensity that closely aligns with most of the options currently available in grocery stores. Traditional consumers also returned positive utility scores for Colby Jack and white Cheddar cheese, along with chipotle peppers and hatch peppers and a medium frequency of peppers. This result suggested that although these consumers preferred HPC characteristics traditionally seen in HPCs such as Pepper Jack, they are open to new varieties of HPC. This group was the most frequent consumers of HPC, with $42 \%$ indicating they consumed HPC at least once per week.

Cluster $2(\mathrm{n}=152)$ designated the mild consumers, as their ideal HPC was a Monterey Jack with small 
pieces of multicolored jalapeno peppers at a medium frequency and medium heat (Figure 2). This group also returned positive utility scores for low spice and burn intensity and had the lowest utility $(-60.7)$ for ghost peppers (an intensely spicy pepper; Bray, 2013). Most mild consumers indicated they generally preferred mild $(16 \%)$ or medium $(58 \%)$ spice and burn intensity when consuming spicy foods. This group had the least frequent users of HPC, as only $32 \%$ of mild consumers reported consuming HPC at least once per week. Cluster $3(\mathrm{n}=183)$ was designated the spicy consumers (Figure 2). These consumers' ideal HPC was a white Cheddar with medium-sized, multicolored pieces of habanero peppers at a medium frequency with a medium spice and burn intensity. These consumers also displayed positive utility for mozzarella cheese (21.0), high frequency of pepper pieces (19.5), and a high burn and spicy intensity (19.4). This group reported the highest frequency of spicy foods consumption, with $75 \%$ of individuals reporting they consume spicy foods at least once per week. However, only $38 \%$ of individuals in the spicy consumers group reported consuming HPC at least once per week. This discrepancy may be due to differences between the ideal HPC for this consumer group and the options currently available to them on the market.

\section{Descriptive Analysis of Hot-pepper Cheeses}

The 2 replications of cheeses were similar in moisture (mean $\pm \mathrm{SD} ; 40 \pm 0.88 \%$ ), fat in $\mathrm{DM}(51 \pm 0.58 \%)$, salt content $(1.8 \pm 0.18 \%)$, and $\mathrm{pH}(5.3 \pm 0.05)$. Similarly, trained panel profiles were not distinct $(P>0.05)$ for cheese replicates, and thus results are reported as the means of both cheese replications. Based on the results observed during the online survey, HPC were made using different combinations of hatch peppers, jalapeno peppers, and habanero peppers to create a range of heat and burn intensity and visible pepper color. The HPC were all characterized by mild, cooked, milky, and whey flavors consistent with young Monterey Jack cheeses (results not shown). As expected, HPC differed in pepper aromatic flavor and hot-pepper burn intensity (Table 2). Pepper burn intensity followed an approximately linear pattern across the $5 \mathrm{HPC}$, with formulation 1 having the lowest pepper burn intensity and formulation 5 having the highest. With the exception of formulation 1, which only used green hatch chile peppers, all cheeses contained multicolored (red and green or red, green, and orange) pepper pieces, as this was preferred by all consumer groups in the online $\mathrm{ACBC}$ exercise.

\section{Consumer-Acceptance Testing}

Consumers had the highest overall liking score for formulations 2,4 , and 5 , which were made with a mix of red and green jalapenos and orange habanero peppers with variable pepper flavor and pepper burn (Tables 2, 3 ). Consumer cheese flavor, saltiness, and texture liking scores for cheeses were not different $(P>0.05$; results not shown). Formulation 1 received the lowest appearance liking among the cheeses. This cheese had only green hatch peppers, further indicating that consumers prefer multicolored pepper pieces in HPC, which was noted by the survey. The cheeses with the highest hotpepper burn by trained panelists (formulations 4 and 5) received the highest spiciness and pepper flavor liking scores from consumers (Figures 3, 4). Overall, consumers preferred HPC with more intense pepper burn over less intense HPC.

Consumer results were segmented using consumer self-reported answers to the same spice and burn preference question used in the online survey. We hypothesized that these groups would perceive actual HPC differently, as there is a wealth of evidence to suggest that consumer liking for capsaicin and pepper burn is not homogeneous. Some consumers dislike any level of capsaicin irritation, whereas others actively seek higher levels of pungency (Dalton and Byrnes, 2016). Researchers have studied this variety in hedonic response to spicy foods and posited several theories about why preference exhibits such variety. It has been documented that sensitivity to capsaicin decreases with age, as younger subjects ( $<40 \mathrm{yr})$ were observed to have significantly lower threshold scores for capsaicin than older subjects (Just et al., 2007). Several studies have observed that frequent and regular users of spicy products consistently indicated the intensity of oral-induced capsaicin burn lower than nonusers (Lawless et al., 1985; Stevenson and Yeomans, 1993; Nolden

Table 2. Mean pepper aromatic and pepper burn intensities from descriptive analysis of hot-pepper cheeses ${ }^{1}$

\begin{tabular}{lcc}
\hline Prototype & Pepper aromatic & Pepper burn \\
\hline Formulation 1 & $2.8^{\mathrm{c}}$ & $0.6^{\mathrm{e}}$ \\
Formulation 2 & $2.8^{\mathrm{c}}$ & $1.3^{\mathrm{d}}$ \\
Formulation 3 & $2.7^{\mathrm{c}}$ & $2.0^{\mathrm{c}}$ \\
Formulation 4 & $3.0^{\mathrm{b}}$ & $3.1^{\mathrm{b}}$ \\
Formulation 5 & $3.7^{\mathrm{a}}$ & $5.7^{\mathrm{a}}$
\end{tabular}

${ }^{\mathrm{a} e}$ Different letters following means within a column indicate significant differences $(P<0.05)$.

${ }^{1}$ Attributes were evaluated using a 0 -to-15 point universal intensity scale with trained judges $(n=7)$ evaluating each cheese replication in duplicate. Most natural cheese flavors fall between 0 and 4 on this scale (Drake et al., 2001). 
and Hayes, 2017). No differences have been observed in sensitivity to capsaicin between genders, but Byrnes and Hayes (2015) studied the effect of gender on spicy food liking and intake of spicy foods and hypothesized that men who consumed spicy foods may be more strongly motivated by extrinsic rewards (sensitivity to reward), whereas women displayed stronger motivation by intrinsic rewards (sensation seeking). It has also been hypothesized that food neophobia, or avoidance of unfamiliar or novel foods, also plays a role in spicy food liking and perception. Törnwall et al. (2014) grouped participants into "basic" eaters and "adventurous" eaters using the food neophobia scale and found that adventurous eaters expressed higher liking for sour and spicy foods and were also less food neophobic (Törnwall et al., 2014). Consumers who indicate they prefer intensely spicy foods enjoy the sensation of pungency and burn (Rozin and Schiller, 1980).

Consumers who reported they preferred mild or medium-spicy foods were classified as "mild" consumers, and participants who preferred hot or extra hot foods were considered "hot" consumers. Self-reported preference for spicy foods did affect some liking attributes of the HPC used in consumer testing (Table 3). No differences between consumer groups were observed for formulation 3 for any attribute, but significant differences in spiciness or pepper burn liking were documented for the other 4 cheeses. As such, mild and hot consumers perceived low burn intensity and high burn intensity cheeses differently, and these cheeses may be polarizing for consumer groups. Mild consumers significantly $(P<0.0001)$ preferred the appearance (as well as spiciness and pepper flavor) of formulation 1 over hot consumers. However, the color difference between formulation 1 (green peppers only) and other cheeses (green-red or green-red-orange) may not have been the sole driver of differences in appearance acceptability. By analysis of JAR scores, $89 \%$ of hot consumers felt that formulation 1 had too few pepper pieces compared with only $67 \%$ of mild consumers (data not shown), which may also contribute to differences in perception between the 2 groups for formulation 1. Pepper color as well as amount of visible peppers may be visual cues to HPC consumers that suggest pepper flavor and spiciness and burn. Formulation 1 scored the lowest $(P<$ 0.05 ) in pepper color liking (mean $=5.5$ on 9 -point scale) and formulations 2 and 4 scored the highest $(P<0.05$; mean $=6.9$ and 7.0 on a 9 -point scale, respectively; results not shown). However, significant differences in pepper color liking were only observed among cheeses. No differences in pepper color liking were observed between consumer groups (hot vs. mild; $P>0.05)$. Positive consumer responses to pepper color and appearance characteristics may also have a halo effect on other HPC characteristics (Lawless and Heymann, 2013).

Penalty analysis of the 5 HPC provided further context to consumer liking (Table 4). Formulations 1 to 4 were penalized for having too few pepper pieces, not being spicy enough, and having too much cheese flavor and not enough pepper flavor. Formulation 4 was penalized for both too little and too much cheese flavor, indicating that consumers felt the cheese-pepper flavor balance was not optimal or there was disagreement among consumers regarding the flavor balance of formulation 4. Further analysis of penalty scores from overall consumers $(\mathrm{n}=196)$, mild consumers $(\mathrm{n}$ $=98)$, and hot consumers $(\mathrm{n}=98$; results not shown $)$ indicated that $20.5 \%$ of hot consumers and $19.5 \%$ of mild consumers felt that formulation 4 had too much cheese flavor and not enough pepper flavor. In contrast, $37.7 \%$ of mild consumers penalized formulation 4 for

Table 3. Consumer liking means for significantly different attributes ${ }^{1}$ of hot-pepper cheeses for all consumers $(\mathrm{n}=196)$ and consumers grouped by self-reported mild versus hot and spicy food preference $(n=98$ in each group)

\begin{tabular}{|c|c|c|c|c|c|c|c|c|c|c|c|c|}
\hline Prototype & \multicolumn{12}{|c|}{ Question } \\
\hline Formulation 1 & $5.5^{\mathrm{C}}$ & $5.8^{\mathrm{a}}$ & $5.1^{\mathrm{b}}$ & $5.6^{\mathrm{B}}$ & 5.8 & 5.4 & $4.0^{\mathrm{C}}$ & $4.7^{\mathrm{a}}$ & $3.4^{\mathrm{b}}$ & $4.2^{\mathrm{C}}$ & $4.9^{\mathrm{a}}$ & $3.6^{\mathrm{b}}$ \\
\hline Formulation 2 & $6.7^{\mathrm{AB}}$ & 6.8 & 6.7 & $6.4^{\mathrm{A}}$ & 6.4 & 6.3 & $5.2^{\mathrm{B}}$ & $5.7^{\mathrm{a}}$ & $4.7^{\mathrm{b}}$ & $5.5^{\mathrm{B}}$ & 5.7 & 5.2 \\
\hline Formulation 3 & $6.6^{\mathrm{B}}$ & 6.6 & 6.7 & $5.9^{\mathrm{B}}$ & 5.7 & 6.0 & $5.2^{\mathrm{B}}$ & 5.3 & 5.1 & $5.2^{\mathrm{B}}$ & 5.4 & 4.9 \\
\hline
\end{tabular}




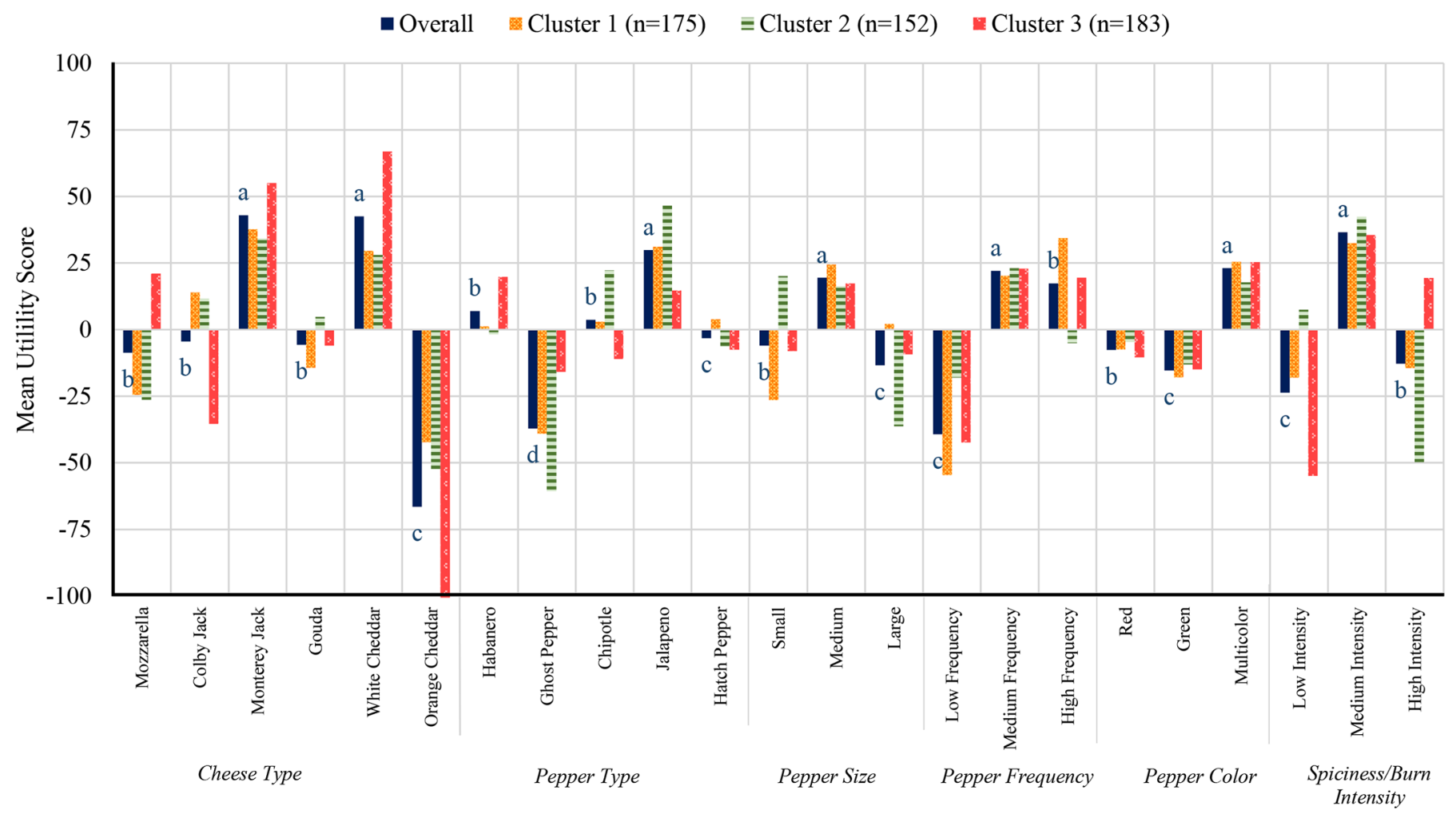

Figure 3. Overall and clustered adaptive choice-based conjoint utility scores for preferred hot-pepper cheese attributes from online survey (n $=510$ ). Utility scores (zero-centered) and higher (more positive) scores are representative of more appealing levels within an attribute, whereas lower (more negative) scores represent less appealing characteristics. Means followed by different letters (a-d) within an attribute are significantly different $(P<0.05)$.

having too much pepper flavor and not enough cheese flavor, whereas only $16.7 \%$ of hot consumers responded in the same manner. This discrepancy between the 2

\section{Formulation 1}

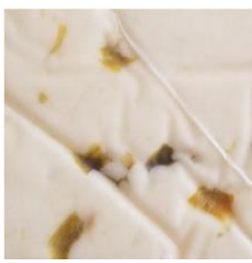

\section{Formulation 4}

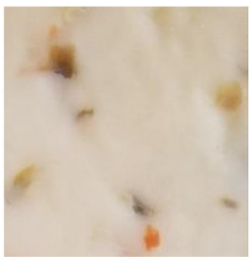

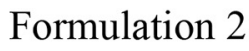
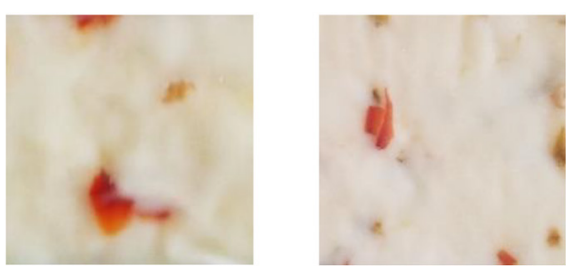

\section{Formulation 5}

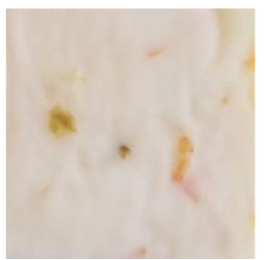

Figure 4. Photos of hot-pepper cheeses used in consumer-acceptance testing. Formulations are described in Table 1. consumer groups likely resulted in formulation 4 being penalized for both too much and too little pepper flavor. Overall, formulation 5 was penalized for being too hot and spicy and having too much pepper flavor. Consumer responses to the spiciness JAR question were also segmented by mild consumers versus hot consumers to better understand how pepper burn intensity was perceived by HPC consumers and to identify an optimal point for hot-pepper burn in HPC (Figure 5). Most consumers ( $57.4 \%$ overall) indicated that formulation 4 was just about right in spiciness, with $50.6 \%$ of mild consumers and $64.1 \%$ of hot consumers selecting this option. Formulation 5 was perceived as JAR by $52.9 \%$ of consumers overall, $39.8 \%$ of mild consumers, and $68.1 \%$ of hot consumers. A pepper burn intensity between 3 and 5 on a 15-point universal intensity scale by trained panelists may be the optimal burn intensity for most HPC consumers. The HPC consumers who enjoy intensely spicy foods may prefer HPC that have pepper burn intensities above this range, but cheese manufacturers risk alienating HPC consumers who prefer mild spice if the burn intensity is too high.

The ideal HPC for HPC consumers from the conjoint analysis [Monterey Jack cheese with medium-sized, 
Table 4. Results of penalty analysis (\%) on hot-pepper cheeses from consumer-acceptance testing $(\mathrm{n}=196 \text { consumers })^{1}$

\begin{tabular}{|c|c|c|c|c|c|c|}
\hline Variable & Level & Formulation 1 & Formulation 2 & Formulation 3 & Formulation 4 & Formulation 5 \\
\hline \multirow{2}{*}{ Amount of pepper pieces JAR } & JAR & 20.6 & 54.8 & 51.3 & 59.4 & 54.8 \\
\hline & Too much & 1.3 & 8.4 & 5.8 & 9.7 & 14.2 \\
\hline \multirow{2}{*}{ Spiciness JAR } & JAR & 12.3 & 30.3 & 32.1 & 57.4 & 52.9 \\
\hline & Too much & 3.2 & 3.2 & 1.3 & 18.7 & $39.4^{*}$ \\
\hline $\begin{array}{l}\text { Cheese flavor to pepper flavor } \\
\text { balance JAR }\end{array}$ & Too little & $78.1^{*}$ & $53.5^{*}$ & $57.1^{*}$ & $20.0^{*}$ & 5.8 \\
\hline
\end{tabular}

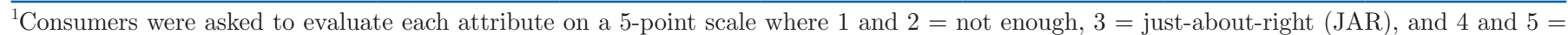
too much.

${ }^{2}$ Scale for cheese flavor to pepper flavor balance JAR was too much cheese flavor (1 and 2) to too much pepper flavor (4 and 5$)$.

*Indicates significant penalty on overall liking $(P<0.05)$.

multicolored (red and green) pieces of jalapeno peppers at a medium frequency and a medium heat and burn intensity] aligned with acceptance test results. Survey participants $(\mathrm{n}=510)$ displayed positive utility scores for both jalapeno and habanero peppers, but significantly preferred jalapenos over habaneros conceptually $(P<0.05)$. This was corroborated by consumer taste test results in which participants $(\mathrm{n}=196)$ also preferred the appearance of cheeses with multicolor peppers (formulations 2, 3, 4, and 5 vs. formulation 1) as well as the flavor of cheeses made with both jalapenos and habaneros (formulations 4 and 5) over a cheese made with only jalapenos (formulation 3; Table 3; Figures 6 and 7). Survey participants also indicated that the presence of both red and green peppers in an HPC was more appealing than one pepper color alone. This result was supported by acceptance testing, as consumers returned the lowest pepper color liking and appearance liking scores for formulation 1 which was made with green hatch chiles. Formulation 4, which was perceived as JAR in spiciness by most taste test participants, was made using a $70 \%$ jalapeno pepper mix to $30 \%$ habanero ratio and had a pepper burn intensity of 3.1 by trained panel profiling. This study only evaluated the perception of HPC by self-reported HPC consumers and did not evaluate the opinions of non-HPC cheese consumers. Therefore, these results are only applicable to current HPC users. The acceptability of HPC with

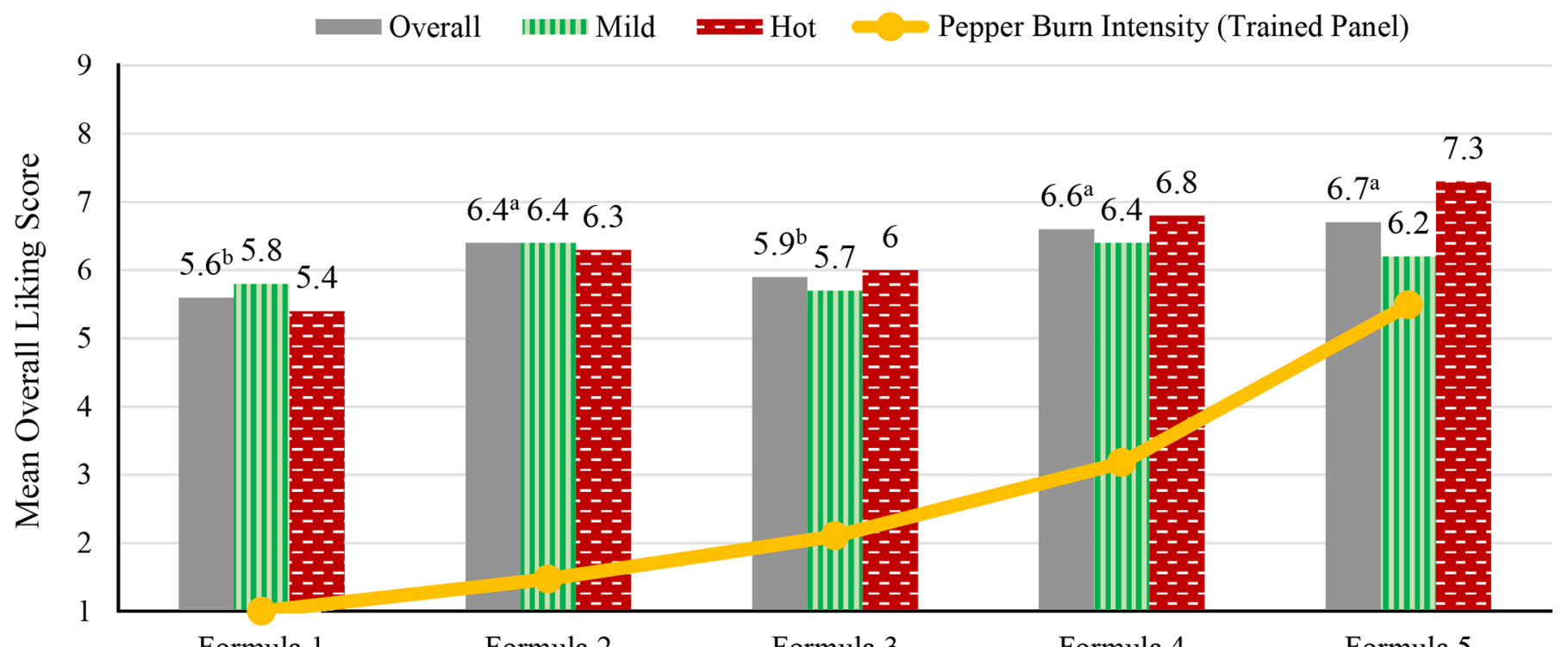

Formula 1

Formula 2

Formula 3

Formula 4

Formula 5

Figure 5. Mean overall liking scores for hot-pepper cheeses from consumer-acceptance testing plotted with pepper burn intensity from the trained panel. Consumers ( $=196$ total) were self-reported into groups designated "mild" ( $=98)$ or "hot" (n = 98) food likers. Different letters $(\mathrm{a}, \mathrm{b})$ indicate significant differences in overall liking $(P<0.05)$. Formulations are described in Table 1. 


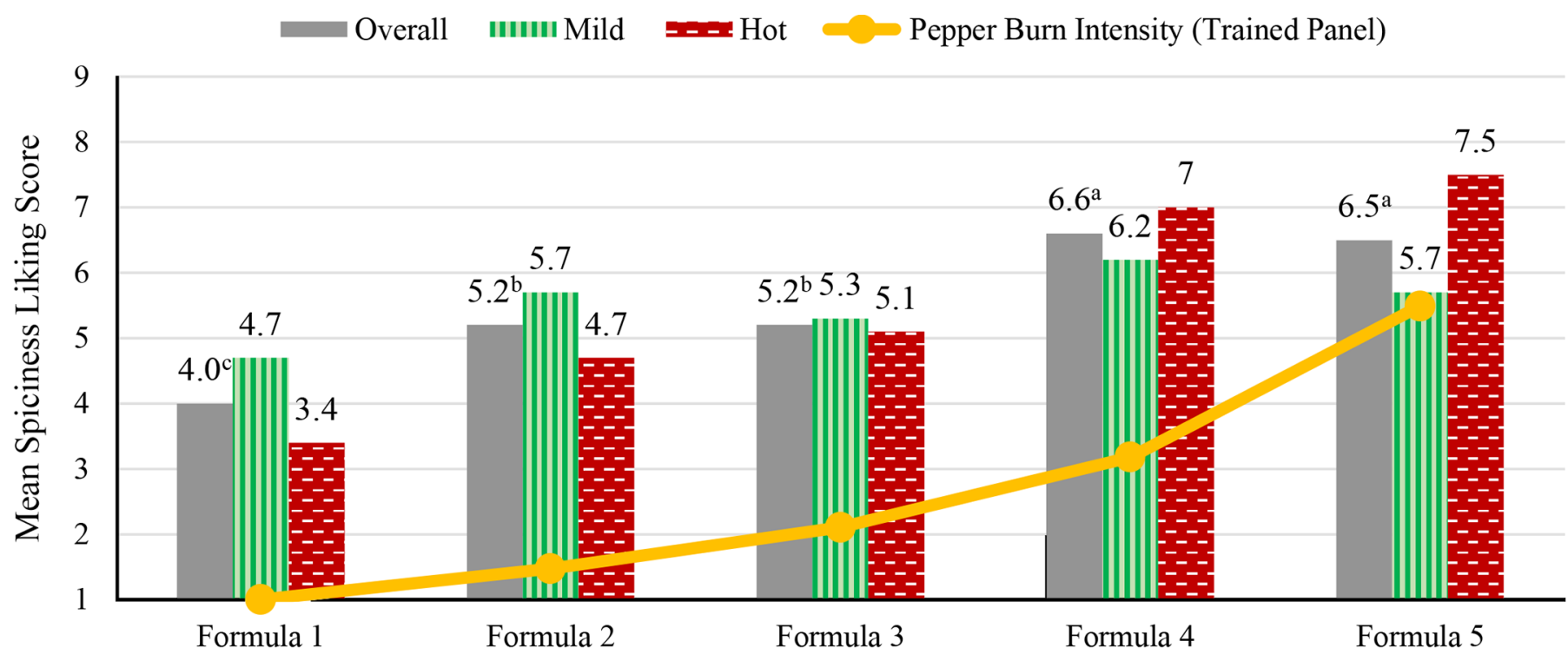

Figure 6. Mean spiciness and burn liking scores for hot-pepper cheeses by consumer group from consumer-acceptance testing plotted with pepper burn intensity from trained panel profiling. Consumers were placed into groups designated "mild" (n $=98)$ or "hot" (n = 98) food likers. Letters indicate significant differences in spiciness liking $(P<0.05)$. Formulations are described in Table 1.

pepper species that have pepper aromatics but low pepper burn, such as hatch chiles (formulation 1), were less acceptable to current HPC users but it is unknown if these cheeses would be appealing to non-HPC users. As such, the appeal of this type of pepper cheese may be underrepresented in the current study. From the conjoint analysis, HPC consumers in this study were also interested in nontraditional pepper cheese ingredients such as chipotle peppers. Cheese manufacturers should consider diversifying their HPC offerings to enforce existing HPC consumer liking of HPC and potentially introduce new users to the category.

\section{CONCLUSIONS}

Results of this study illustrated the characteristics of HPC that consumers value and helped to identify the ideal HPC that appeals to consumers. The consumer

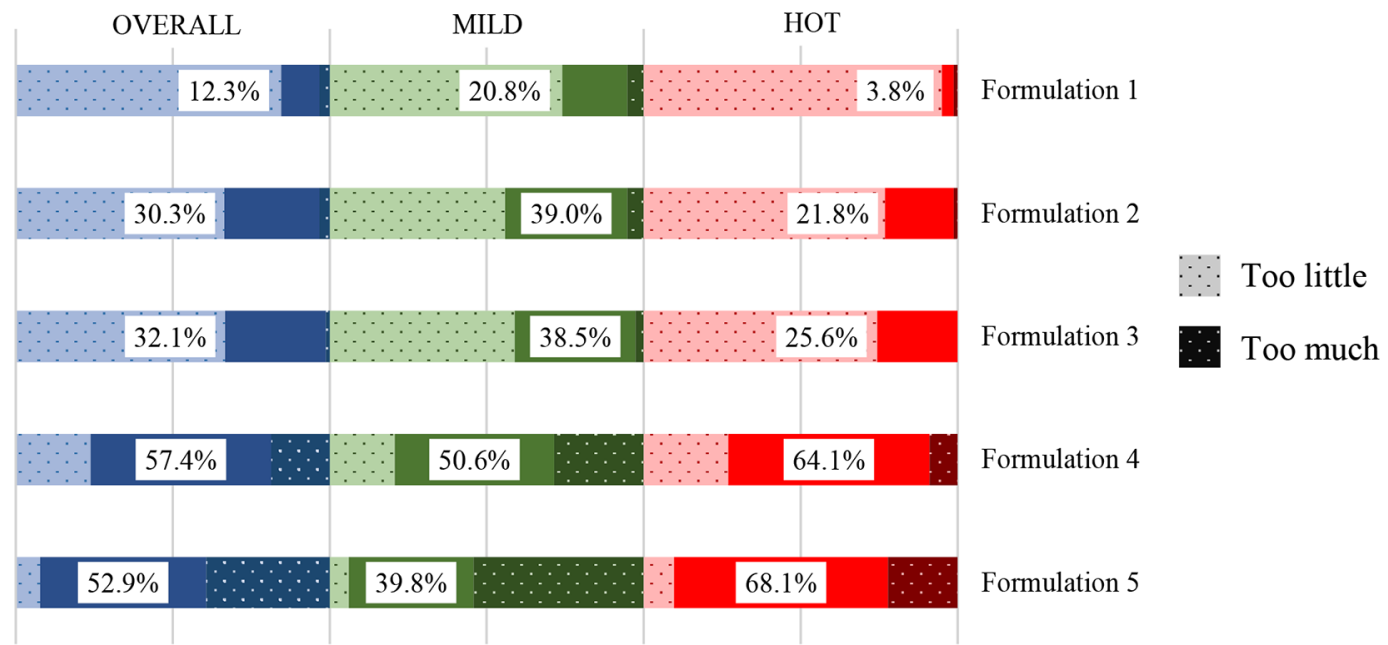

Figure 7. Segmented results for just-about-right (JAR) question percentages for spiciness of hot-pepper cheese consumers. Consumers were asked to evaluate cheese spiciness and hotness attribute on a 5 -point scale where 1 and $2=$ not spicy or hot enough, $3=\mathrm{JAR}$, and 4 and $5=$ too spicy or hot. Percentages represent percent of consumers in each group (overall $n=196$, mild consumers $n=98$, hot consumers $n=98$ ) that indicated the sample was JAR. Formulations are described in Table 1. 
conceptual ideal HPC was a Monterey Jack cheese with medium-sized, multicolored (red and green) pieces of jalapeno peppers at a medium frequency and a medium heat and burn intensity. Consumers consistently demonstrated preference for multicolored pepper pieces in HPC. General spice level and burn intensity preferences affected liking of spiciness and pepper flavor in HPC. Consumers who self-reported that they preferred mild or medium intensity products were more accepting of lower burn intensity HPC, whereas consumers who selfreported preference for higher burn intensity products showed higher acceptance for HPC with higher pepper burn intensity. A hot-pepper burn intensity between 3 and 5 on a trained panel universal intensity scale may be the optimal burn intensity for most current HPC consumers, but consumers who enjoy intensely spicy foods may find HPC that have pepper burn intensities above this range attractive as well.

\section{ACKNOWLEDGMENTS}

Funding provided in part by Dairy West (Meridian, ID), National Dairy Council (Rosemont, IL), and Glanbia Nutritionals (Twin Falls, ID). The authors are grateful to Luke Lundberg (Glanbia Nutritionals, Twin Falls, ID) for assistance. The use of tradenames does not imply endorsement nor lack of endorsement by those not mentioned. The authors have not stated any conflicts of interest.

\section{REFERENCES}

Allison, A. A., E. Chambers, E. Gibson, and F. M. Aramouni. 1999. Sensory characteristics of heat-processed and fresh tomato salsa containing honey. J. Food Sci. 64:560-564. https://doi.org/10 .1111/j.1365-2621.1999.tb15085.x.

Anonymous. 2019. Spicy Perceptions. Accessed May 27, 2021. https: //info.kalsec.com/hubfs/Consumer_Trends/Kalsec_eBook _SpicyPerceptions_1019.pdf.

Anonymous. 2021. Kano Analysis. Accessed May 19, 2021. https:// sapioresearch.com/kano-analysis.

Bray, M. 2013. Ghost Pepper Guide: Heat, Flavor, Uses. Accessed May 20, 2021. https://www.pepperscale.com/ghost-pepper/.

Byrnes, N. K., and J. E. Hayes. 2015. Gender differences in the influence of personality traits on spicy food liking and intake. Food Qual. Prefer. 42:12-19. https://doi.org/10.1016/j.foodqual.2015 .01 .002 .

Carden, L. A., M. P. Penfield, and A. M. Saxton. 1999. Perception of heat in cheese sauces as affected by capsaicin concentration, fat level, fat mimetic and time. J. Food Sci. 64:175-179. https://doi .org/10.1111/j.1365-2621.1999.tb09886.x.

Chen, L.-F. 2012. A novel approach to regression analysis for the classification of quality attributes in the Kano model: an empirical test in the food and beverage industry. Omega 40:651-659. https://doi .org/10.1016/j.omega.2011.12.004.

Cliff, M., and H. Heymann. 1992. Descriptive analysis of oral pungency. J. Sens. Stud. 7:279-290. https://doi.org/10.1111/j.1745-459X .1992.tb00195.x.

Colonna, A., C. Durham, and L. Meunier-Goddik. 2011. Factors affecting consumers' preferences for and purchasing decisions re- garding pasteurized and raw milk specialty cheeses. J. Dairy Sci. 94:5217-5226. https://doi.org/10.3168/jds.2011-4456.

Dalton, P., and N. Byrnes. 2016. Psychology of Chemesthesis - Why Would Anyone Want to Be in Pain? John Wiley \& Sons Ltd.

Del Toro-Gipson, R. S., P. V. Rizzo, D. J. Hanson, and M. A. Drake. 2021. Consumer perception of smoked Cheddar cheese. J. Dairy Sci. 104:1560-1575. https://doi.org/10.3168/jds.2020-18711.

Djekic, I., J. Ilic, R. P. F. Guiné, and I. Tomasevic. 2020. Can we understand food oral processing using Kano model? Case study with confectionery products. J. Texture Stud. 51:861-869. https://doi .org/10.1111/jtxs. 12550 .

Drake, M. A., S. C. Mcingvale, P. D. Gerard, K. R. Cadwallader, and G. V. Civille. 2001. Development of a descriptive language for Cheddar cheese. J. Food Sci. 66:1422-1427. https://doi.org/10 $.1111 /$ j.1365-2621.2001.tb15225.x.

Guzmán, I., and P. W. Bosland. 2017. Sensory properties of chile pepper heat - and its importance to food quality and cultural preference. Appetite 117:186-190. https://doi.org/10.1016/j.appet.2017 .06 .026 .

Harwood, W. S., and M. A. Drake. 2018. Identification and characterization of fluid milk consumer groups. J. Dairy Sci. 101:8860-8874. https://doi.org/10.3168/jds.2018-14855.

Jervis, S. M., J. M. Ennis, and M. A. Drake. 2012. A comparison of adaptive choice-based conjoint and choice-based conjoint to determine key choice attributes of sour cream with limited sample size. J. Sens. Stud. 27:451-462. https://doi.org/10.1111/joss.12009.

Just, T., H. W. Pau, S. Steiner, and T. Hummel. 2007. Assessment of oral trigeminal sensitivity in humans. Eur. Arch. Otorhinolaryngol. 264:545-551. https://doi.org/10.1007/s00405-006-0218-4.

Kamp, K. 2020. Cheese - US - November 2020. Accessed Jun. 14, 2021. https://reports-mintel-com.prox.lib.ncsu.edu/display/987264/.

Kano, N., N. Seraku, F. Takahashi, and S.-I. Tsuji. 1984. Attractive quality and must-be quality. Journal of the Japanese Society for Quality Control 14:147-156.

Kim, M. K., K. Lopetcharat, and M. A. Drake. 2013. Influence of packaging information on consumer liking of chocolate milk. J. Dairy Sci. 96:4843-4856. https://doi.org/10.3168/jds.2012-6399.

Lawless, H., P. Rozin, and J. Shenker. 1985. Effects of oral capsaicin on gustatory, olfactory and irritant sensations and flavor identification in humans who regularly or rarely consume chili pepper. Chem. Senses 10:579-589. https://doi.org/10.1093/chemse/10.4 .579 .

Lawless, H. T., and H. Heymann. 2013. Context effects and biases in sensory judgement. Pages 216-217 in Sensory Evaluation of Food: Principles and Practices. Second Edition. D. R. Heldman, ed. Springer Science \& Business Media.

McLean, K. G., D. J. Hanson, S. M. Jervis, and M. A. Drake. 2017. Consumer perception of retail pork bacon attributes using Adaptive Choice-based Conjoint Analysis and Maximum Differential scaling. J. Food Sci. 82:2659-2668. https://doi.org/10.1111/1750 $-3841.13934$.

Meilgaard, M., G. V. Civille, and B. T. Carr. 2007. The spectrum descriptive analysis method. Pages 189-253 in Sensory Evaluation Techniques. 4th ed. CRC Press.

Moskowitz, H. 2001. Creating new product concepts for foodservice - the role of conjoint measurement to identify promising product features. Food Serv. Technol. 1:35-52. https://doi.org/10.1046/j .1471-5740.2001.00004.x.

Nolden, A. A., and J. E. Hayes. 2017. Perceptual and affective responses to sampled capsaicin differ by reported intake. Food Qual. Prefer. 55:26-34. https://doi.org/10.1016/j.foodqual.2016.08.003.

Oltman, A. E., K. Lopetcharat, E. Bastian, and M. A. Drake. 2015. Identifying key attributes for protein beverages. J. Food Sci. 80:S1383-S1390. https://doi.org/10.1111/1750-3841.12877.

Orme, B. K. 2006. Getting Started with Conjoint Analysis: Strategies for Product Design and Pricing Research. Research Publishers.

Ponnam, A., D. Sahoo, and M. Balaji. 2011. Satisfaction-based segmentation: Application of Kano model in Indian fast food industry. Journal of Targeting, Measurement and Analysis for Marketing 19:195-205. https://doi.org/10.1057/jt.2011.20. 
Reyes-Escogido, M. de L., E. G. Gonzalez-Mondragon, and E. Vazquez-Tzompantzi. 2011. Chemical and pharmacological aspects of capsaicin. Molecules 16:1253-1270. https://doi.org/10 $.3390 /$ molecules 16021253 .

Rizzo, P. V., W. S. Harwood, and M. A. Drake. 2020. Consumer desires and perceptions of lactose-free milk. J. Dairy Sci. 103:69506966. https://doi.org/10.3168/jds.2019-17940.

Rotar, L. J., and M. Kozar. 2017. The use of the Kano model to enhance customer satisfaction. Organizacija 50:339-351. https://doi .org/10.1515/orga-2017-0025.

Rozin, P., and D. Schiller. 1980. The nature and acquisition of a preference for chili pepper by humans. Motiv. Emot. 4:77-101. https:// doi.org/10.1007/BF00995932.

Schiano, A. N., W. S. Harwood, P. D. Gerard, and M. A. Drake, 2020. Consumer perception of the sustainability of dairy products and plant-based dairy alternatives. J. Dairy Sci. 103:11228-11243. https://doi.org/10.3168/jds.2020-18406.

Schlossareck, C., and C. F. Ross. 2019. Electronic tongue and consumer sensory evaluation of spicy paneer cheese. J. Food Sci. 84:15631569. https://doi.org/10.1111/1750-3841.14604.

Shahbandeh, M. 2020. Per Capita Consumption of American Cheese in the U.S., 2019. Accessed Nov. 19, 2020. https://www.statista.com/ statistics/183803/per-capita-consumption-of-american-cheese-in -the-us-since-2000/.

Speight, K. C., A. N. Schiano, W. S. Harwood, and M. A. Drake. 2019. Consumer insights on prepackaged Cheddar cheese shreds using focus groups, conjoint analysis, and qualitative multivariate analysis. J. Dairy Sci. 102:6971-6986. https://doi.org/10.3168/jds .2018-16209.

Stevenson, R. J., and M. R. Yeomans. 1993. Differences in ratings of intensity and pleasantness for the capsaicin burn between chili likers and non-likers; implications for liking development. Chem. Senses 18:471-482. https://doi.org/10.1093/chemse/18.5.471.

Törnwall, O., K. Silventoinen, T. Hiekkalinna, M. Perola, H. Tuorila, and J. Kaprio. 2014. Identifying flavor preference subgroups. Genetic basis and related eating behavior traits. Appetite 75:1-10. https://doi.org/10.1016/j.appet.2013.11.020.

\section{ORCIDS}

C. M. Racette () https://orcid.org/0000-0003-4934-6021

M. A. Drake $\odot$ https://orcid.org/0000-0002-4744-2493 